\title{
MAGNETRON DRIVEN CLASSICAL MICROTRON AS AN INJECTOR FOR A WIDE BAND TUNABLE COMPACT FAR INFRARED FREE ELECTRON LASER
}

\author{
Grigori M. Kazakevitch ${ }^{\mathrm{a}, *}$, Young Uk Jeong ${ }^{\mathrm{a}}$, Byung Cheol Lee ${ }^{\mathrm{a}}$, Sung Oh Cho ${ }^{\mathrm{a}}$, Jongmin Lee ${ }^{\mathrm{a}}$, \\ Victor P.Belov ${ }^{\mathrm{b}}$, Nikolai G. Gavrilov ${ }^{\mathrm{b}}$. \\ ${ }^{a}$ Lab. for Quantum Optics, Korea Atomic Energy Research Institute, P.O.Box 105, Yusong, Taejon, \\ 305-600, Rep. Korea. \\ ${ }^{b}$ Budker Institute of Nuclear Physics RAS, Academician Lavrentyev 11, Novosibirsk, 630090, \\ Russia.
}

\begin{abstract}
A compact 12 turn classical microtron driven by a 2.8 $\mathrm{GHz}$ magnetron has been improved for use as an injector of a compact wide-band far infrared (FIR) free electron laser (FEL). The microtron provides an accelerated beam current of up to $70 \mathrm{~mA}$ by total energy of up to $7.2 \mathrm{MeV}$ in a $5.5 \mu$ s duration macro pulse. The frequency of the magnetron was stabilized in the range of $(3-4) \times 10^{-5}$ by the frequency pulling of the magnetron with the reflected wave from the accelerating cavity. The measured energy spread and emittances in vertical and horizontal components of the electron beam were less than $0.4 \%, 1.5$ $\mathrm{mm} \cdot \mathrm{mrad}$ and $3.5 \mathrm{~mm} \cdot \mathrm{mrad}$, respectively. A measured temporal deviation of the bunch repetition rate of less than $120 \mathrm{kHz}$ could be obtained by optimization of the microtron operating conditions for the FEL. With a total energy of electrons and macro pulse current of $7 \mathrm{MeV}$ and $45 \mathrm{~mA}$ respectively stable FIR lasing could be obtained in the wavelength range from 97 to $150 \mu \mathrm{m}$ by changing the undulator $\mathrm{K}$-parameter. The accelerating cavity moving system was upgraded to increase the variable range of the electron beam energy from 4.3 to 7 $\mathrm{MeV}$, which provides wide tuning range of the FIR FEL wavelength up to $300 \mu \mathrm{m}$.
\end{abstract}

\section{Microtron magnetic-vacuum system.}

A 12-turn microtron-FIR FEL injector developed on the basis of its prototype [1] has an unusual $750 \mathrm{~mm}$ diameter and $267 \mathrm{~mm}$ high chamber-less magnetic-vacuum system in which the vacuum volume is composed of magnet poles and the back magnet yoke. Magnet coils are sealed with extended surface of magnet poles to back yoke by turning from the same metal block. Thus only one indium wire seals the magnetic-vacuum system of the microtron as whole.

\footnotetext{
*Corresponding author. Current Postal address: Lab. for Quantum Optics, Korea Atomic Energy Research Institute, P.O. Box 105, Yusong, Taejon, 305-600, Rep. Korea.

Tel: +82 42868 8253; fax: +8242861 8292;

E-mail: kaza@kaeri.re.kr

Permanent Postal address: Budker Institute of Nuclear Physics,

Akademician Lavrentyev, 11, Novosibirsk, 630090, Russia.
}

Different sizes of microtron magnetic system were calculated by varying the thickness, location, shape of shims, coils geometry and insets cross section to get the maximal diameter of vacuum space with a magnetic field inhomogeneity approximately $0.1 \%$. Measured under microtron operating conditions (the magnetic field was in the range of $0.9-1.1 \mathrm{kG}$ ) the value of the magnetic field inhomogeneity for 12 turns was less than $0.2 \%$.

The beam-extracting channel was made from low carbon steel. A compensation system having low carbon steel pivots positioned above and below the extracting channel was used to avoid magnetic field distortion near the entrance of the channel. The extraction efficiency measured under this condition was approximately $90 \%$.

\section{RF - system of microtron.}

The microtron RF-system was designed to provide the intra-pulse frequency stability for FIR FEL operation. A $2.8 \mathrm{GHz}, 2.5 \mathrm{MW}$ pulse power MI-456A magnetron fabricated in Russia was chosen as the RF generator. The wave-guide line of the microtron (Fig. 1 (a)), including a ferrite valve with back losses approximately $15 \mathrm{~dB}$ by 2 MW pulse power, a directional coupler for measurements of the forward and reflected waves and vacuum waveguide window, was optimized for size to serve at the same time for stabilization of the magnetron frequency by the frequency pulling.

The microtron RF-system including the magnetron and the wave-guide line ending with the accelerating cavity was assembled as a whole on a moving frame and was sealed as part of a vacuum system by a flexible bellow to provide a $178 \mathrm{~mm}$ shift of the accelerating cavity inside the vacuum volume. This shift permits us to change the number of microtron orbits and provides the range of the electron beam energy variation acceptable for the wide-band-tunable FIR FEL.

The cylindrical microtron cavity with an oscillation mode $\mathrm{E}_{010}$ and coupling coefficient $\beta_{0}=5.4$ working in the first type of acceleration [2] was made from oxygen-free copper with diamond turning of the internal surface. 


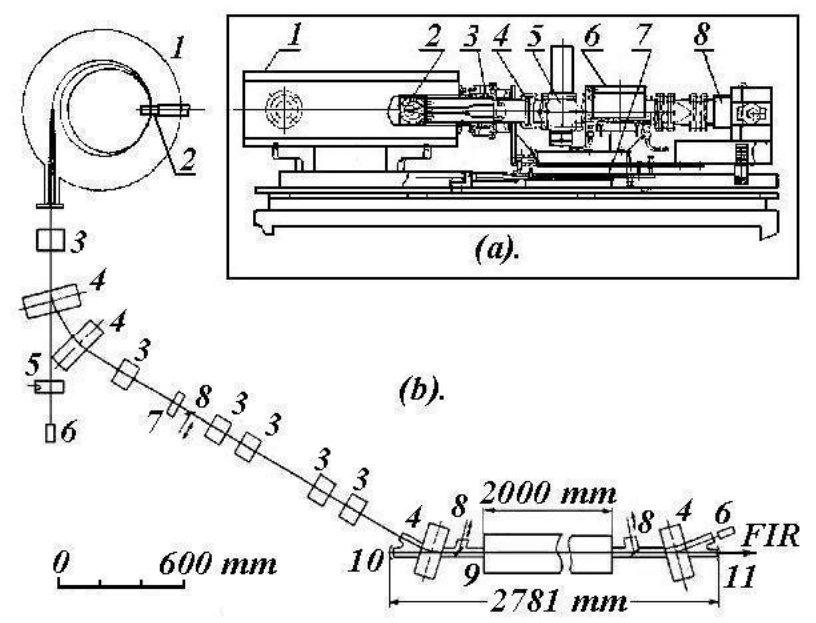

Fig. 1. 12-turn microtron-injector of the FIR FEL facility.

(a). General view of the microtron RF system.

1-Microtron magnet, 2-accelerating cavity, 3-bellow, 4wave-guide vacuum window, 5-directional coupler, 6ferrite valve, 7-moving frame, 8-magnetron.

(b). Layout of the FIR FEL based on the microtroninjector.

1-Microtron, 2-accelerating cavity, 3-quadrupole lens, 4bending magnet, 5-straight-flight measuring cavity, 6beam dump, 7-beam current transformer, 8-OTR-screen, 9-U-25 undulator, 10-full mirror, 11-coupling mirror.

The Q-factor of the unloaded cavity was approximately $10^{4}$. The controlled water-cooling system keeps the temperature of the cavity stable during microtron operation in the range of $15 \mathrm{C}^{0}-50 \mathrm{C}^{\mathrm{o}}$ with accuracy approximately $\pm 1 \mathrm{C}^{\circ}$.

A $\mathrm{LaB}_{6}$ [100]-face monocrystalline $2.5 \mathrm{~mm}$ in diameter cathode heated by a tungsten filament was used in the microtron as an emitter of electrons [3]. The cathode unit as a whole was precisely fixed at the cover of the accelerating cavity. The measured capture coefficient for the optimal position of the emitting surface was in the range of $4.5-6.8 \%$ for a total energy of accelerated electrons approximately $7 \mathrm{MeV}$ and macro pulse current in the range of 50-70 mA. Under these conditions the measured cathode brightness temperature was approximately $1600 \mathrm{C}^{\circ}$ [3] and a significant part of the emission current was generated by the work of the cathode in a strong electric field due to the Shottky effect. Corresponding to these conditions the average cathode lifetime is at least $1000 \mathrm{~h}$ and is limited mainly by the emitter evaporation.

For investigation of the bunch repetition rate stability during the macro pulse a low Q-factor straight-flight measuring cavity was mounted in the straight section of the beam line as shown in Fig. 1(b). The RF signals arising by the pass of electron bunches through the measuring cavity have been analyzed with the heterodyne method [4]. From the measured data we could choose operating conditions of the RF system providing successful operation of the FIR FEL. The average inaccuracy of the bunch repetition rate deviation measurements of $(3-5) \times 10^{-6}$ could be obtained for the 100 ns time interval.

\section{Microtron modulator.}

In the linear magnetron modulator a 20-cells storage line was used, comprised by a tunable $25-\mathrm{Ohm}$ impedance 5cells charging lines type PL-02 with a hydrogen thyratron type TGI1-1000/25, high voltage (HV) rectifier and resonant charging circuit. Dumping circuits were used for protection against back high voltage breakdown by the random discharge in the magnetron. A thyristor submodulator controlled by the CAMAC master timer provides start up the thyratron. The start up of the submodulator is synchronized with zero level crossings of the microtron cathode AC filament current to avoid distortion of the trajectory of electrons emitted from the cathode.

The high voltage of the HV-rectifier filtering capacity $\left(C_{f}\right)$ has been stabilized by a $25 \mathrm{kHz}$ inverter feeding the $\mathrm{HV}$ rectifier. The inverter, controlled by a precise comparator, provides fast and exact charging of the $C_{f}$ up to the required value of the high voltage with a two steps mode of charging. Under this condition the portion of the $C_{f}$ charge is maximal at the beginning of process, but then, around the required value of the filtering capacity voltage, the charging portion is minimal. The stability of the storage line voltage by such a method of charging is approximately $0.1 \%$ [5].

The modulator was mounted inside the microtron support as a unit with a size of $1800 \times 600 \times 800 \mathrm{~mm}^{3}$.

\section{Experimental investigation of microtron parameters.}

Bunching property of the microtron has been investigated by numerical simulation of the electron beam longitudinal motion in 2-D geometry with real size of cathode. The simulation results based on the measured bunch repetition rate deviations show qualitative agreement with those of the coherent radiation measurements by the electron beam passing through an undulator [6].

Main parameters of the microtron have been measured by the extraction of the accelerated beam from 12-th orbit, the total energy approximately $7 \mathrm{MeV}$ with macro pulse current of 45-55 mA. Under these conditions the emission current was in the range of 0.9-1.25 A and had growth in the range of $0.25-0.35 \mathrm{~A}$ which was caused by bombardment of the cathode surface with back returning electrons during pulse durations of RF-power of more than $6 \mu \mathrm{s}$. Compensation for the accelerating field abatement caused by growth of the emission current was provided by linear growth of the cavity feeding RF-power in the range approximately $100 \mathrm{~kW}$. This was achieved by the final tuning of the modulator charging line, which provided approximately $10 \%$-growth of the magnetron current during the macro pulse. This provided a relatively 
flattop to the macro pulse current in spite of a sufficient macro pulse duration by injection from the internal cathode as shown in Fig. 2.

The electron frequency drift of the magnetron caused by deviation of the magnetron current during the macro pulse was significantly suppressed by frequency pulling because of a standing wave feeding the magnetron. For more effective frequency pulling the microtron operating parameters were chosen to provide a minimal initial value of magnetron-accelerating cavity detuning by acceptable for FIR lasing value of accelerated current.

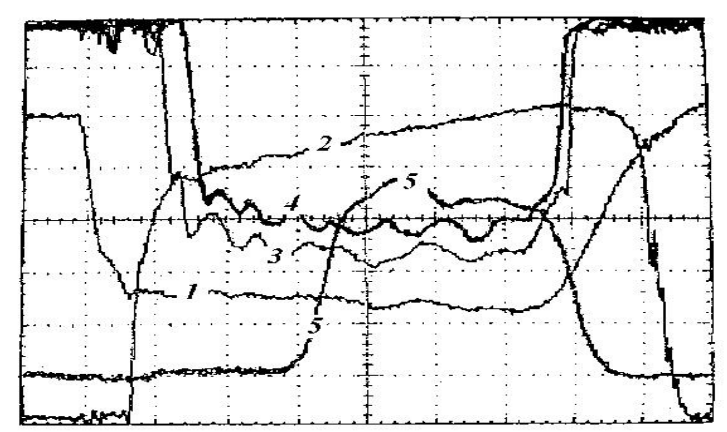

Fig. 2. Oscillograph records of signals by microtron parameters optimized for FIR lasing.

1-magnetron current, vert. scale is $22.2 \mathrm{~A}$ /div. 2-emission current, vert. scale is $200 \mathrm{~mA} /$ div. 3-accelerated current, vert. scale is $10 \mathrm{~mA} /$ div. 4-current at the undulator entrance, vert. scale is $10 \mathrm{~mA} /$ div. 5-FIR lasing signal at the output of liquid He cooled $\mathrm{Ge}-\mathrm{Ga}$ detector, vert. scale is $1 \mathrm{~V} / \mathrm{div}$. The horizontal scales always are $1 \mu \mathrm{s} / \mathrm{div}$.

By initial magnetron-accelerating cavity detuning in the range of $160-180 \mathrm{kHz}$ the value of accelerated current was up to $55 \mathrm{~mA}$. Under this condition the bunch repetition rate had growth during macro pulse with a constant approximately $20 \mathrm{kHz} / \mu \mathrm{s}$ that corresponded to the frequency stabilization coefficient $\sim 10$. For the value of the initial detuning approximately $140 \mathrm{kHz}$, the corresponding maximal value of the accelerated current was up to $45 \mathrm{~mA}$, and the intra-pulse bunch repetition rate deviations were in the range of $\sim 100 \mathrm{kHz}$. Note that deviations of the bunch repetition rate had an oscillating character. Measured average amplitude of the frequency modulation was approximately $30 \mathrm{kHz}$ with a period of oscillation in the range of 0.6-0.7 $\mu$ s [4].

The energy spread and emittance of the electrons were determined from the measurements of the beam profile sizes depending on quadrupole lens current using a movable $0.1 \mathrm{~mm}$ thick polished aluminum Optical Transition Radiation (OTR) screen located after the first quadrupole lenses doublet, Fig. 1(b). The measurements accuracy was approximately $100 \mu \mathrm{m}$. Obtained data of the energy spread, vertical and horizontal emittances are at most $0.4 \%, 1.5 \mathrm{~mm} \cdot \mathrm{mrad}$ and $3.5 \mathrm{~mm} \cdot \mathrm{mrad}$ respectively.

\section{Use of the microtron as an injector for a FIR FEL.}

The microtron was used as an injector for the FIR FEL. The accelerated beam extracted from the microtron was matched by the beam line, Fig. 1(b), to transport the electrons through approximately $3 \mathrm{~m}$ long wave-guide chamber with an internal cross section of $2 \times 30 \mathrm{~mm}^{2}$. The chamber was disposed in the gap of a variable magnetic field U-25 undulator. The vertical and horizontal sizes of the matched electron beam at the undulator entrance were $0.6 \mathrm{~mm}$ and $2.1 \mathrm{~mm}$, respectively. A loss of the electron beam transported through the undulator was insignificant by the variation of undulator magnetic field from minimum to maximum. Cylindrical gold-coated mirrors covered both ends of the wave-guide chamber to form a confocal free space mode in horizontal plane and a waveguide mode in vertical plane of the FIR resonator. By changing the undulator K-parameter in the range of 1.051.6, and setting the total energy of accelerated electrons and the macro pulse current at the entrance of the waveguide chamber of $7 \mathrm{MeV}$ and $40 \mathrm{~mA}$ respectively, we successfully detected FIR FEL generation with deep saturation tunable in the range of wavelength $97-150 \mu \mathrm{m}$. The measured radiated macro pulse power was $30-50 \mathrm{~W}$ by the coupling coefficient of FIR resonator $~ 1.7 \%$ [7]. The oscillograph record of the attenuated FIR lasing signal with a wavelength of $120 \mu \mathrm{m}$ is shown in Fig. 2.

\section{References}

[1] G.M. Kazakevich, V.N. Marusov, G.I. Silvestrov. "8 $\mathrm{MeV}$ microtron-the injector for an electron synchrotron." Proceed. of second Asian Symposium on Free Electron Lasers, BINP, Novosibirsk, 1995.

[2] S.P. Kapitza and V.N. Melekhin. The Microtron. London. Harwood, 1978.

[3] G.I. Kuznetsov. "A Gun for the Microtron.” Instrum. and Experim. Techniques, V 40, No.3, (1997), 424.

[4] G.M. Kazakevitch, Y.U .Jeong et al. "Start up of lasing in compact far infrared Free Electron Laser driven by $8 \mathrm{MeV}$ Microtron." in Accelerator-based Sources of IR and Spectroscopic Applicat. G.L. Carr, P. Dumas,Editors. Proc. of SPIE, V. 3775, 71, 1999.

[5] G.M. Kazakevich, V.V. Rashenko, Y.F. Tokarev. "Linear Modulator of the Microtron." Instrum. and Experim. Techniques, V 42, No. 3, (1999), 365.

[6] G.M. Kazakevitch, S.S. Serednyakov et al. "Bunching Properties of a Classical Microtron-Injector for a Far Infrared Free Electron Laser." $22^{\text {nd }}$ Free Electron Laser Conference, FEL 2000, Duke University, 2000.

[7] Y.U. Jeong, G.M. Kazakevitch, et al. "First lasing of the KAERI Compact Far-Infrared Free-Electron Laser Driven by a Conventional Microtron." $22^{\text {nd }}$ Free Electron Laser Conference, FEL 2000, Duke University, 2000. 